\title{
Human Modeling for Efficient Predictive Collision Detection
}

\author{
Gabriel Streitmatter \\ University of Florida
}

Faculty Mentor: Gloria Wiens, Department of Mechanical and Aerospace Engineering

\begin{abstract}
As demands on manufacturing rapidly evolve, flexible manufacturing is becoming more essential for acquiring the necessary productivity to remain competitive. An innovative approach to flexible manufacturing is the introduction of fenceless robotic manufacturing. This involves operations in which a human and a robot share a space to complete tasks together. Such operations, however, pose serious safety concerns. Before Human Robot Collaboration can be implemented, robots must be capable of safely operating within dynamic environments. The robot must be able to do this quickly during online operation. This paper outlines an algorithm for predictive collision detection. This algorithm gives the robot the ability to look ahead at its trajectory, and the motion in its environment and predict potential collisions. The algorithm approximates a continuous swept volume of any articulated body along its trajectory by taking only a few time sequential samples of the predicted orientations of the body and creating surfaces that patch the orientations together with Coons patches. Run time data collected on this algorithm indicate that the algorithm can accurately predict future collisions in under $30 \mathrm{~ms}$.

Keywords: Predictive Collision Detection, Swept Volume Interference, Coons Patches
\end{abstract}

\section{Introduction}

Automation of manufacturing processes over the past few decades has yielded enormous benefits in efficiency and quality. This was accomplished by increasing speed and precision, and improving material handling devices with sensory capability. Products now are experiencing quicker life cycles and increased customization. Manufacturing processes must be more flexible to meet rapidly changing demands. This can be accomplished by developing human-robot collaboration (HRC) methodologies; taking advantage of the speed, power, and precision of robots as well as the creativity and adaptability of humans (Kruger et al., 2009). To do this, robots must be flexible enough to operate safely around humans. This further elevates challenges as safety concerns surround unrestricted operation of robots near humans. Before HRC can be implemented, robots must learn to avoid collisions with humans.

In the authors previous work, an algorithm for predictive collision detection was developed to enable a robot to forecast its motion amongst moving ellipsoid objects to identify collisions. 
Coons patches, a computer graphics technique for defining surfaces, were leveraged to approximate the robot's position throughout time as a swept volume (Streitmatter and Wiens, 2020). In this paper, the algorithm is extended to model the articulated, multi-link body trajectories of the human to predict collisions between a robot and human. The goal of the paper is to evaluate the posit that highly accurate and computationally cheap collision checks can be performed by modeling both the human and the robot with Coons patches. This paper contributes to a multi-university/industry/national laboratory collaborative research effort to develop HRC techniques for sensing of dynamic environments, forecasting change in the environment, and enabling robots to respond safely and productively (Yin et al., 2018). The algorithm in this paper will contribute to an HRC Proactive Adaptive Collaborative Intelligence (PACI) module used to control robot motion and operation (Nicora et al., 2020).

\section{Related Work}

One of the simplest and fastest ways of performing predictive collision detection is to employ Multiple Interference Detection. In this process a representative set of configurations throughout the robot's trajectory are checked for interference. If, however, the sampling frequency is too low, collisions can be missed. Another more robust approach is Swept Volume Interference in which an object is swept along its trajectory to create a composite shape representing the total volume affected by the object. A collision can be identified by overlaying the position and time of the object at all points along its trajectory and identifying where spatial and temporal data intersect (Jiménez et al., 2001). This is computationally expensive and difficult to implement in real time. For online operation of a robot in dynamic environments in which human safety is in question, fast and efficient algorithms and techniques are needed.

One combination of both approaches is employed in (Mainprice \& Berenson, 2013), where a pre-existing 3D surface is sampled as it moves along its trajectory at a high frequency such that an approximate swept volume is created. While this approach is faster than computing a swept volume analytically, it requires the pre-existence of a 3D surface, and a large number of samples to construct the surface.

Another point cloud-based attempt creates a model of the sweeps by sensing all points on the human and robot and predicting all occupied points over time with an RGB-D flow algorithms. Doing this for every point however is computationally burdensome, and the algorithm proved to not execute in real time, with a maximum frequency of 6 to $8 \mathrm{~Hz}$ (Herman et al., 2015). 
The algorithm presented in this paper addresses the need for a computationally faster collision detection algorithm that can operate on much more basic predictions of the human's location and orientation throughout time. By extending the authors prior work to implementing Coons patches to interpolate the human's position throughout time, fewer samples are required to construct a representative swept surface, greatly improving the computational efficiency.

\section{Methods}

The developed algorithm takes as input the predicted joint angles of a human's articulated multi-link body at various instants in time throughout its motion. From this input, forward kinematics techniques are used to define boundary points outlining the human at each predicted instant in time. These boundaries are then used to create a boundary surface that represents the swept volume of the human. This approach is able to strike a tradeoff between Swept Surface Interference and Multiple Detection Interference methods by interpolating between a lower frequency sampling to create a continuous surface. Furthermore, since the swept volume is described only by the boundary surface, points within the surface do not need to be characterized, greatly reducing computer memory and computational costs. For collision detection, only the surface needs to be modeled as it can be assumed that if another volume passes into the space of the swept volume, it must first go through and collide with the swept boundary. The same approach is then applied to the robot. Simulations of the robot and human in a shared workspace are used to evaluate effectiveness and computational efficiency.

\section{Overview of the Approach}

A preliminary grid size must be set to determine coarseness of the grid on which the human and robot swept volumes are to be compared. A fine grid size will result in an increase in computational time but will yield more accurate representation of the swept volume. After each swept volume is created, all points defining the swept volume are relocated to the nearest grid location. This grid allows for direct comparison between multiple swept volumes. The remainder of the algorithm executes three general steps. 


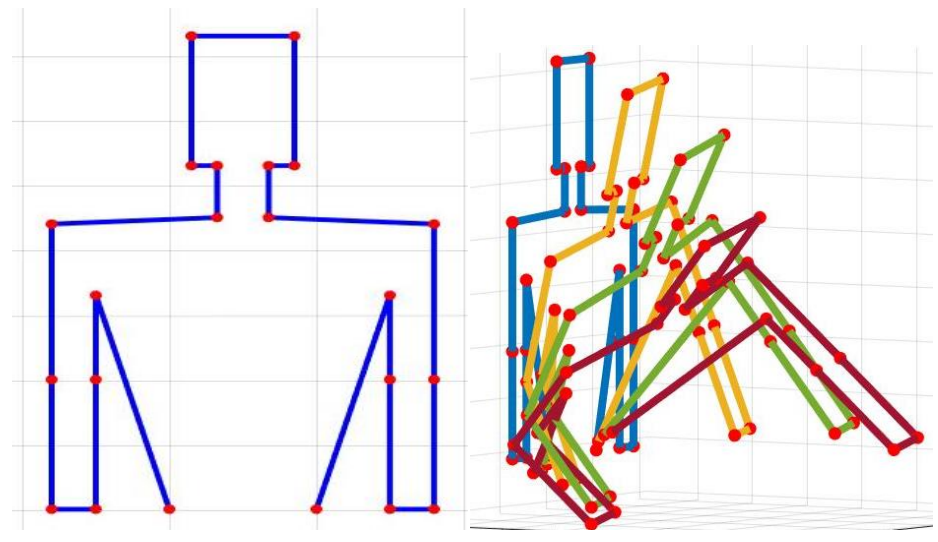

Figure 1. Location of joint offsets (red) determined with forward kinematics.

First, as shown in Figure 1, boundary points (joint offsets, shown in red) are created around each of the joints. These locations are determined directly from the input joint angles through forward kinematics and are positioned such that they create a boundary around the human in a plane facing the direction normal to the initial orientation of the human. As seen in Figure 1, the normal direction would be out of the page. The initial orientation is a consequence of how the coordinate systems used to model each joint are defined. To account for motion in the orthogonal direction, boundary points are also defined in a plane orthogonal to the initial orientation of the human, creating a side profile of the human (Figure 2). This defines a 3-D boundary of the human at one instance in time, such that as each link of the human moves, it will have a section of thickness orthogonal to the direction of motion. This process is repeated for each instance in time for which data on the human is given. To account for uncertainty in future predictions and safety considerations, safety factors are built into the dimensions of the boundary curves such that the boundary curves grow with time. The growth is a function of the body part of the human. For example, since a collision with the head is of greater safety concern than the arm, the dimensions of the head will expand more rapidly than those of the arm.

Next, discrete Coons patches (Farin \& Hansford, 1999) are used to define surfaces between each orientation throughout time. The patches provide an efficient expression for generating point clouds that fill the gaps between the given predicted positions of the human. These patches are key to maximizing computational efficiency by eliminating the need for completing forward kinematics at a high sampling frequency. 


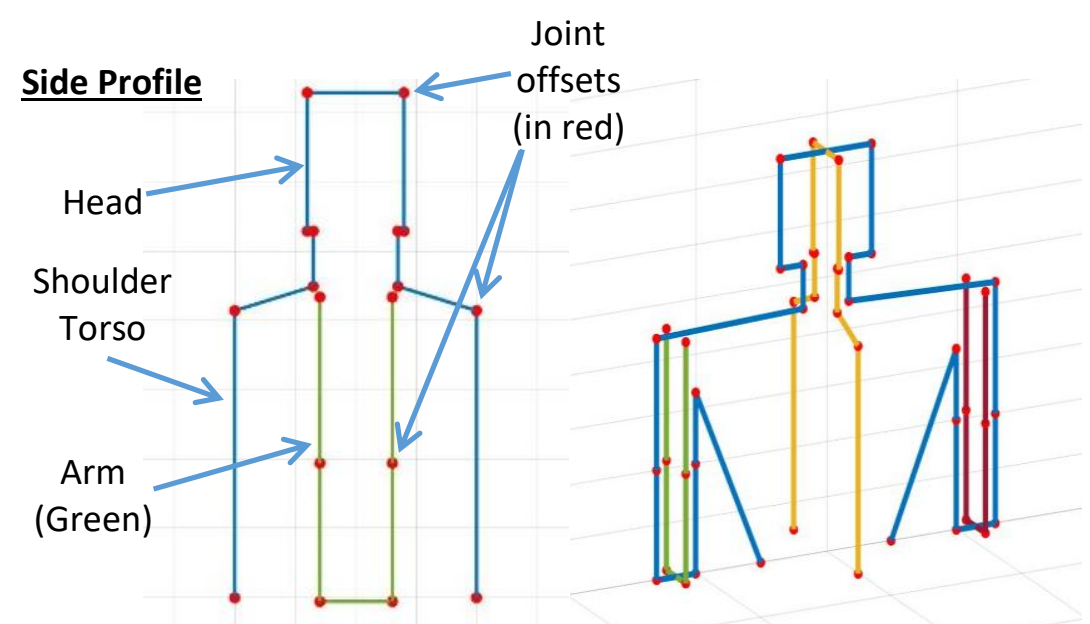

Figure 2. Joint offsets (red) in the orthogonal direction. The right figure shows the overlaid normal and orthogonal curves.

As the human moves, the normal and orthogonal curves will sweep out. As can be seen from Figures 1 and 2, this will still leave portions of the human in the initial and final poses unmodeled. To address this and to create a closed surface therefore completing the volume definition, initial and final conditions of the human are implemented to generate surfaces at the beginning and end of the sweep. This step wraps a surface around the overall swept volume of the human. The resulting swept volume can finally be compared with other swept volumes to identify spatiotemporal intersection of the volumes.

\section{Definition of the Boundary Curves}

The simulated testing utilized in this paper and in the overall project at large is completed with a multi-axis collaborative tabletop robot arm. Because such robots are largely used for small, tabletop assembly and inspection applications, the human model selected to interface with such a robot is a seated worker. Thus, only the waist up of the human was modeled. The model was defined by a torso joint with three axes of rotation, two shoulder joints with three axes of rotation each, two elbow joints with one axis of rotation each, and neck joint with three axes of rotation. Coordinate systems are established with their origin locations fixed at the center of each joint. The location of these systems with respect to each other and with respect to an overall fixed system are described with forward kinematics. Specifically, transformation matrices are used to describe relative distances between two systems, and rotation matrices are used to rotate the systems about their joints. These matrices are defined in Denavit Hartenberg notation. 
To locate the position in the fixed coordinate system of any joint after arbitrary rotations about each joint, a series of transformation and rotation matrices are used to travel from the fixed system through each transformation and rotation of each successive system up to the joint of interest. Consider a point, $P$, with a known position in the coordinate system $n,{ }^{n} P$. To locate ${ }^{n} P$ in the coordinate system of the previous joint, $n-1$, the point must be pre-multiplied by a translation matrix, ${ }_{n}^{n-1} T$, that describes points in system $n$ as seen from system $n-1$. A rotation about the joint in either system will also affect the location of the point. To account for rotations, and a rotation matrix rotating about joint $n, R_{n}$, must also be pre-multiplied. A generalized formula for translating a point in the $n$ system through an arbitrary number of translations and rotations is presented in (1) where ${ }_{1}^{f} T$ is the translation from system one to the fixed system and ${ }^{f} P$ is the location of point $P$ as seen from the fixed system.

$$
{ }^{f} P={ }_{1}^{f} T \quad R_{1}\left[\prod_{i=1}^{n-1}{ }_{i+1}^{i} T R_{i+1}\right]{ }^{n} P
$$

Using this approach, the supplied joint angles for each of the human's joints can be used to find the location of each joint in Cartesian space. Additionally, to provide physical dimension to the human's body, boundary points about each of the human's joints can be defined in the coordinate system of the joint they correspond to and be translated to the fixed frame. To build in a factor of safety and account for uncertainty in the predicted location of the human over time, the distance between these boundary points and the joint they correspond to is increased by the product of a scaling factor and the amount of time between the prediction and the execution time of the algorithm. As rotations occur about each joint, these boundary points and the subsequent points in the kinematic chain also rotate. The left side of Figure 1 depicts the boundary points (marked in red) connected with blue lines. Repeating this process for each step in time, boundary points are defined for the entire trajectory, as seen in the right side of Figure 1, where each instance in time is represented by a different color outline.

To account for the component of motion in the orthogonal direction, a similar boundary is drawn to model the side profile of the human's arm (drawn in green) and torso and head (both drawn in blue) (Figure 2). The interface between the normal and orthogonal boundary curves is also shown (Figure 2 right-side). In the same approach applied above, the boundary points for this profile are defined at each time step.

\section{Application of Coons Patches to Connect the Boundary Curves}


With boundary curves defined for each time step, the next step is to generate surfaces swept between them using the method of discrete Coons patches. Figure 3 illustrates a Coon patch generated as the head travels between two different orientations. The boundary curves defined previously must now be put into a format that the discrete Coons patch formulation applies to. Example boundary curves for a Coons patch are shown in Figure 3. Four boundary curves are used to define each patch (Figure 3 left). The first boundary curve (Curve 1) follows a line between a point on the human at an instant in time and the same point at the next (second) instance in time. The second boundary curve (Curve 2) follows a line between the first point on the human at the second instance in time and a second point on the human at the second instance in time. The third boundary curve (Curve 3) follows a line between the second point at the second instance in time and the same point on the human at the first instance in time. The fourth boundary curve (Curve 4) follows a line between the second point at the first instant in time and the first point at the same instance in time.

With the corner points specified, the curves can be put into the proper mathematical form for application of Coons patches. The mathematical form is shown in Eq. (2), where $\boldsymbol{b}_{0,0}$ to $\boldsymbol{b}_{0, u}$ represents discrete values along Curve $1 ; \boldsymbol{b}_{0, u}$ to $\boldsymbol{b}_{v, u}$ represents Curve $2 ; \boldsymbol{b}_{v, u}$ to $\boldsymbol{b}_{v, 0}$ represents Curve 3; and $\boldsymbol{b}_{v, 0}$ to $\boldsymbol{b}_{0,0}$ represents Curve 4. The variables $v$ and $u$ define the horizontal and vertical components of the mesh of the Coons patch. Higher values of $v$ and $u$ result in more densely packed Coons patches. Finer mesh sizes will lead a more densely defined surface, and better collision detection, but will increase computational time.

$$
[\boldsymbol{b}]=\left[\begin{array}{ccc}
\boldsymbol{b}_{0,0} & \cdots & \boldsymbol{b}_{0, u} \\
\vdots & \ddots & \vdots \\
\boldsymbol{b}_{v, 0} & \cdots & \boldsymbol{b}_{v, u}
\end{array}\right]
$$

Through careful selection of $v$ and $u$, computational effort can be drastically reduced without sacrificing any accuracy. This is done by selecting a minimum $v$ and $u$ such that the maximum distance between any two points in the Coons patch is smaller than the grid size selected at the start of the algorithm. Denser Coons patches will be mapped to the spatial grid size, so high- 
density patches will eventually be generalized by patches that fit the grid size in the final comparison of the swept volumes.

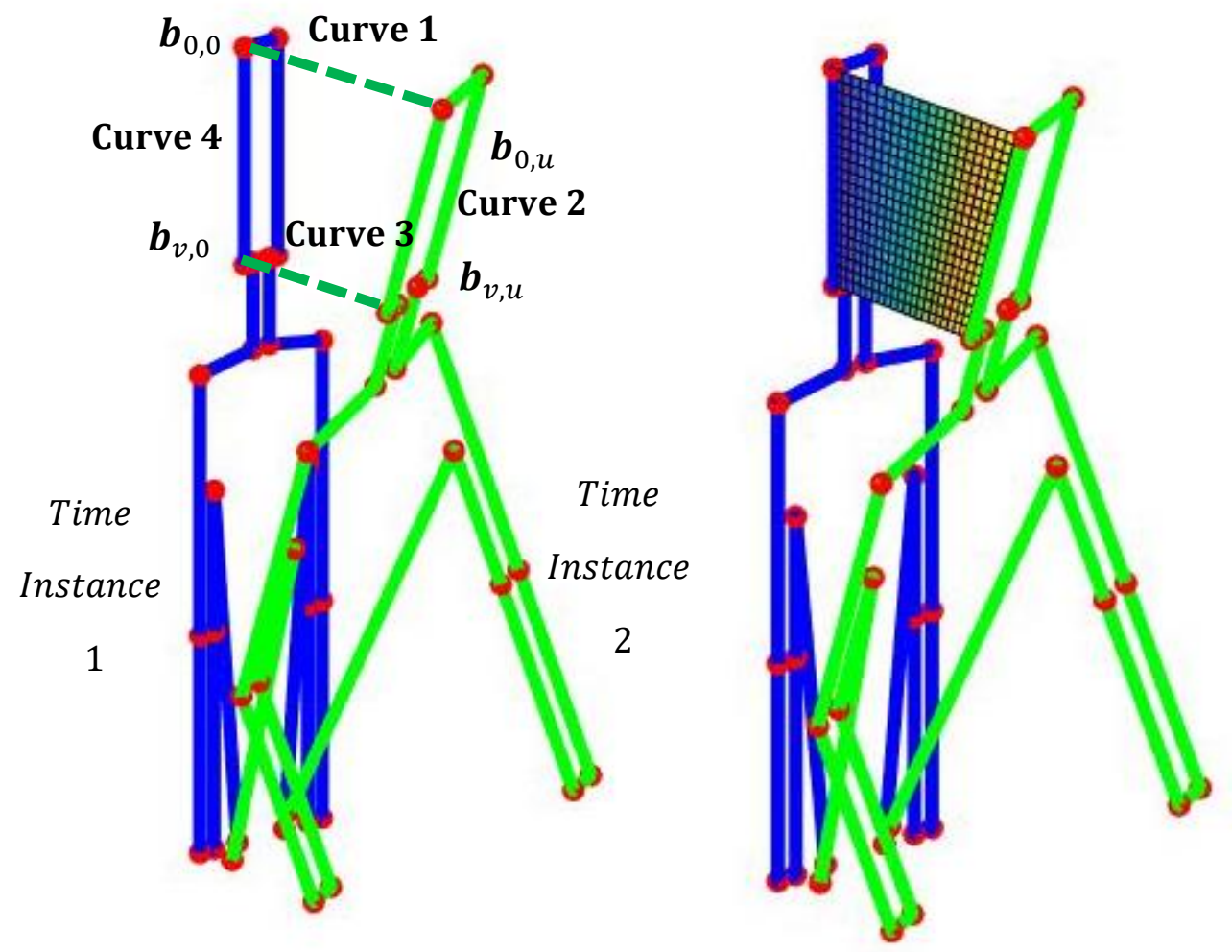

Figure 3. Definition of boundary curves and generation of a patch

Since it is advantageous to uniquely determine $v$ and $u$ for each patch, once the boundary curves have been evaluated, a function looks at their total lengths. The $u$ is determined by evaluating the lengths of Curves 1 and 3. The $u$ is set to equal the length of the longer curve divided by half the grid size. Similarly, $v$ is set by evaluating the lengths of Curves 2 and 4 and dividing the larger of the two by half the grid size. In this way, it is ensured that the Coons patch can be accurately mapped to the grid size without much change in location of the points within the patch and with no risk of marking as unoccupied grid locations that should be occupied.

To define the patch, let $i$ and $j$ represent intermediate values between the boundary curves. The points that lie at each set of indices $i$ and $j$ in $\boldsymbol{b}_{i j}$, where $i$ and $j$ range from 1 to $v-1$ and 1 to $u-1$ respectively, are calculated in the discrete equation of a Coons patch, Eq. (3). Each patch 
contains a discretized grid of points. Each point represents the weighted average of the closest points on the boundary curves with respect to the distance between the point and each curve.

$$
\begin{aligned}
& \boldsymbol{b}_{i, j}=(1-i / v) \boldsymbol{b}_{0, j}+i / v \boldsymbol{b}_{v, j}+ \\
& (1-j / u) \boldsymbol{b}_{\boldsymbol{i}, \mathbf{0}}+j / u \boldsymbol{b}_{\boldsymbol{i}, \mathbf{u}}-[1-i / v \quad i / v]\left[\begin{array}{ll}
\boldsymbol{b}_{\mathbf{0}, \mathbf{0}} & \boldsymbol{b}_{\mathbf{0}, \boldsymbol{u}} \\
\boldsymbol{b}_{\boldsymbol{v}, \mathbf{0}} & \boldsymbol{b}_{v, \boldsymbol{u}}
\end{array}\right]\left[\begin{array}{c}
1-j / u \\
j / u
\end{array}\right]
\end{aligned}
$$

This calculation yields a $(v \times u)$ set of points within the boundary curves. A patch is made for the X, Y, Z, and time dimensions. For the time dimension patch, Curves 2 and 4 lie between two points known at two times. Curves 1 and 3 are linear interpolations between the time at the first configuration and the time at the second, as depicted by example in Figure 3. Applying this process to each segment of the human's motion between each known pose defines the surface of the volume swept by the human. In Figure 4, the X, Y, and Z patches are used to plot the human's swept volume, and the time patch characterizes each point.

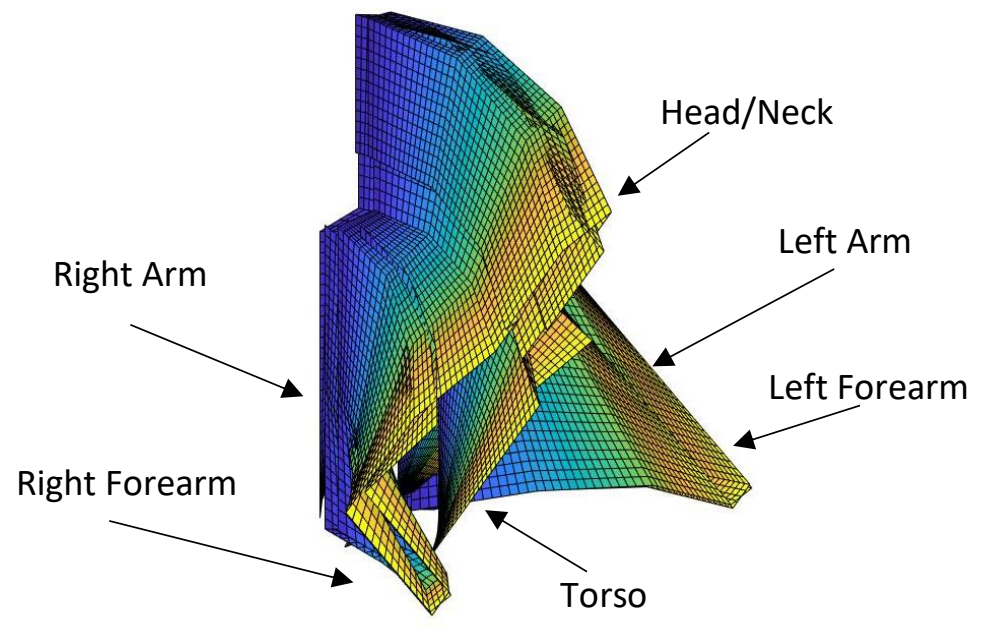

Figure 4. Iterative application of Coons patches to define the surface. Time shown as color.

\section{Setting Initial and Final Conditions}

To close the surface forming the swept volume, Coons patches at the initial and final pose conditions are employed. However, the patches are generated to connect all components of one pose. This is illustrated in Figure 5 for the initial and final pose of the human's motion. 

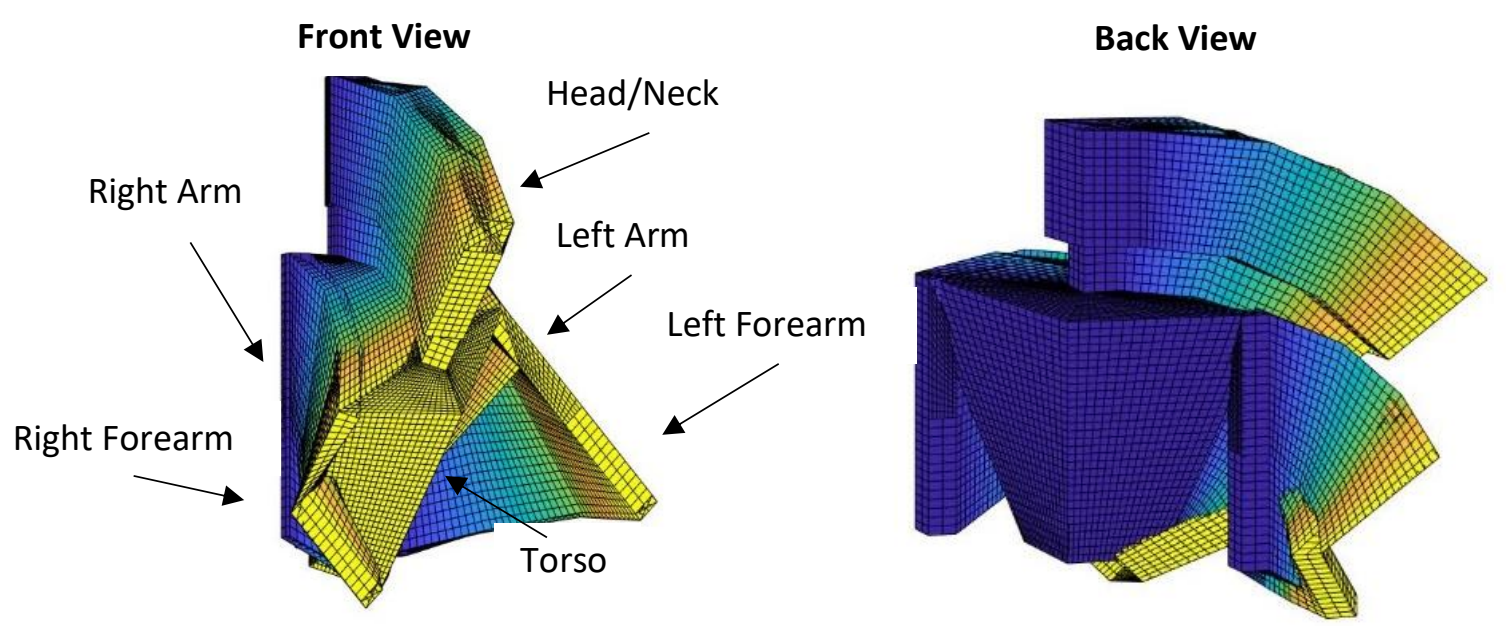

Figure 5. Initial (Left) and final (Right) conditions implemented to close boundary surface

Using this methodology, a robot was modeled. Figure 6 (left) shows the robot in its initial and final orientations, and Figure 6 (right) shows the swept volume. This swept volume is defined in the same fixed system as the human's swept volume.
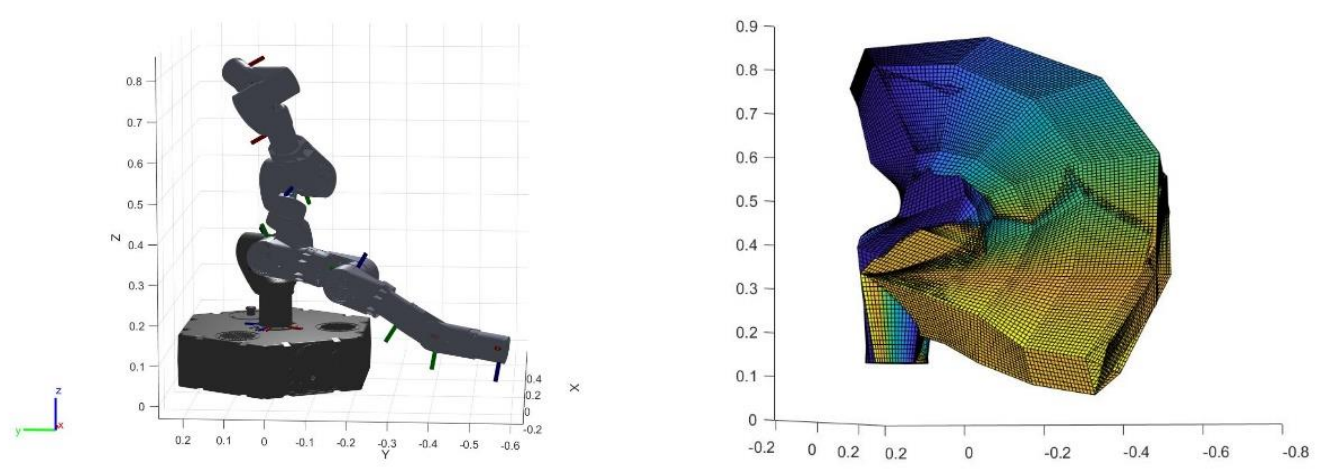

Figure 6. Model of the robot's swept volume. The start and end orientations of the robot are shown (Left).

The swept are mapped into the previously mentioned standardized grid and a standard time array. Each point is fit to the nearest grid space. The time associated with each point is fit to the nearest time. Collisions occur wherever both sweeps contain the same positional and time data. An example collision is shown in Figure 7, where collision points are indicated in red. 


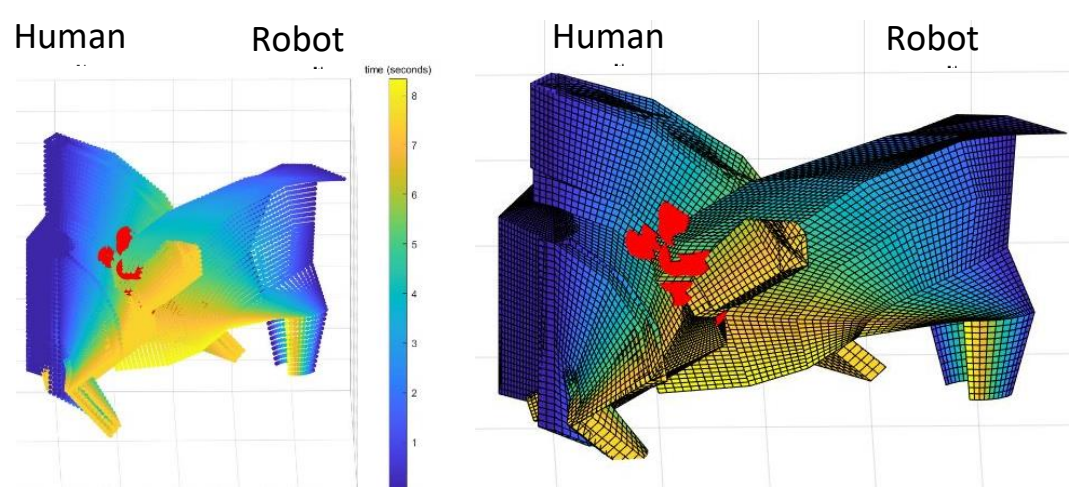

Figure 7. Collision detected between the robot and human swept volumes

\section{Evaluation}

The algorithm was implemented on an Intel ${ }^{\circledR}$ Core $^{\mathrm{TM}}$ i7-7500 CPU processor. Timed tests were run at grid sizes between $0.01 \mathrm{~m}$ to $0.15 \mathrm{~m}$. At each grid size the algorithm was run 100 times to evaluate 100 randomly generated human and robot trajectories. The computational times at each grid size were averaged (Figure 8). An exponential increase in computation occurred for grid sizes below $0.02 \mathrm{~m}$. For grid sizes above $0.07 \mathrm{~m}$, the algorithm runs in less than $30 \mathrm{~ms}$.

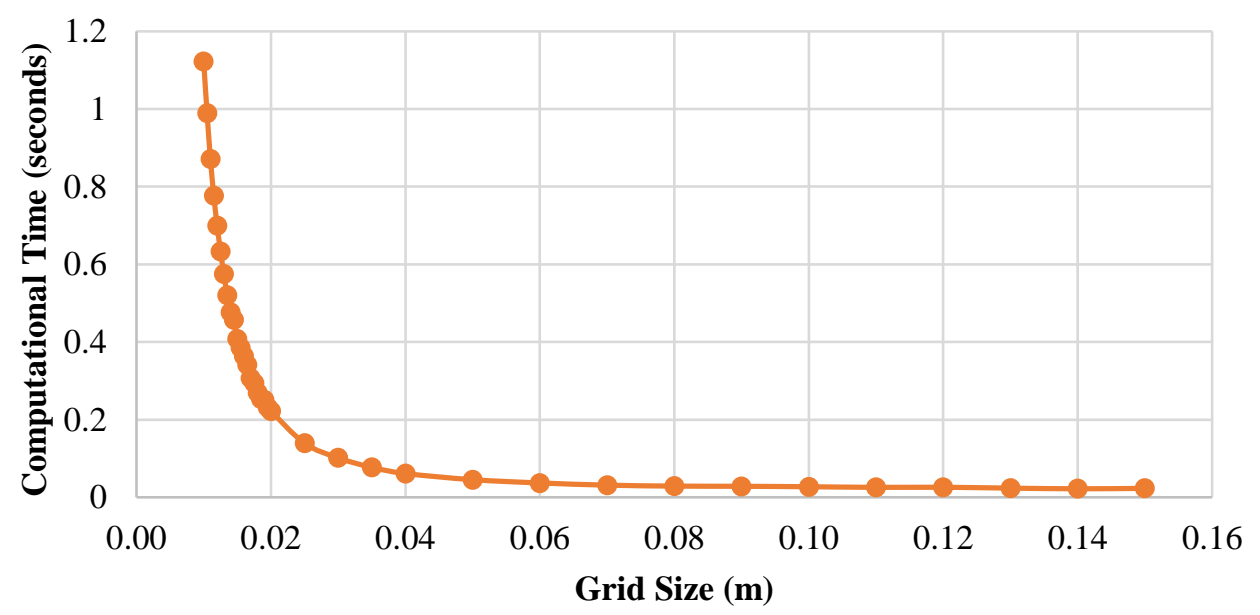

Figure 8. Computational time at each grid size. Time increases exponentially for grids with a spacing of less than 2 $\mathrm{cm}$.

The effectiveness of the algorithm at various grid sizes was evaluated for 100 test cases with random trajectories for the human and robot. Each test case was evaluated at fifteen grid sizes equally spaced between $0.01 \mathrm{~m}$ and $0.15 \mathrm{~m}$. Given that the modeled human, from waist up has a height of $0.73 \mathrm{~m}$, these grid sizes constitute a range of roughly $1 \%$ and $20 \%$ of the total height. The goal of each test was to identify whether there was a collision or not. Of these 100 scenarios, 87 tests maintained the same prediction for all grid sizes. For 8 tests, lower resolution 
iterations misidentified a collision, but converged to a consistent solution for higher resolution grid sizes. The highest resolution at which the algorithm misidentified a collision was at $0.060 \mathrm{~m}$, while the average grid size at which the algorithm converged was $0.110 \mathrm{~m}$. There were four cases in which the algorithm did not to converge to a solution. In these 4 cases, as well as the previously discussed 8 cases, both swept volumes came indistinguishably close to each other, and collisions could not be determined upon visual inspection.

These results indicate that the algorithm is successful in identifying collisions for grid sizes less than $0.06 \mathrm{~m}$. The algorithm can be expected to perform accurate collision detection for grid sizes of $0.100 \mathrm{~m}$ and smaller in less than $30 \mathrm{~ms}$.

\section{Conclusion}

The contribution of this paper is a quick, effective predictive collision detection algorithm designed to run in real time as a background operation of a robot controller. The novelty of the approach was to use Coons patches to interpolate between sampled orientations of a body throughout time. In this way, continuous collision detection is implemented with minimal computation. This algorithm is able to model an entire predicted trajectory and complex articulated obstacles with online computational speed. Integration with its controller, the robot can be intelligently responsive to variations in human movements in a collaborative environment.

Future works will include optimizing grid size according to the input trajectory to accurately model features with the largest grid size possible and implementing the algorithm with sensor data from the human and robot. Finally, the resolution will be improved by narrowing the workspace to the crucial sections. This would allow for much finer detail in the modeled objects.

\section{Acknowledgements}

Funding was provided by University of Florida's Scholars Program. Research conducted in support of NSF/NRI: INT: COLLAB: Manufacturing USA: Intelligent Human-Robot Collaboration for Smart Factory (Award I.D. \#:1830383). Any opinions, findings and conclusions or recommendations expressed are those of the researchers and do not necessarily reflect the views of the National Science Foundation. 


\section{References}

Kruger, J., Lien, T. K., and Verl, A. (2009). Cooperation of Human and Machines in Assembly Lines. CIRP Journal of Manufacturing and Technology, 58(2), 628-646.

Streitmatter, G., and Wiens, G. (2020, July). Human-Robot Collaboration: A Predictive Collision Detection Approach for Operation within Dynamic Environments. Paper presented at the International Symposium on Flexible Automation, Chicago, IL.

Yin, Z., Leu, M., Wiens, G., and Gao, R. (2018, Oct.). "NRI: INT: COLLAB: Manufacturing USA: Intelligent Human-Robot Collaboration for Smart Factory”, 2018 NSF/National Robotics Initiative (NRI) Principal Investigators' Meeting, Arlington, VA, Oct. 29-30. poster (invited).

Nicora, M.L., Ambrosetti, R., Wiens, G.J., and Fassi, I. (2020, June). Human-Robot Collaboration in Smart Manufacturing: Robot Reactive Behavior Intelligence. ASME Manufacturing Science and Engineering Conference, Cincinnati, OH, 8pp..

Jiménez, P., Thomas, F., and Torras, C. (2001). 3D Collision Detection: A Survey. Computers \& Graphics, 25(2), 1-7.

http://graphics.stanford.edu/courses/cs164-09-spring/Handouts/paper_colldect_survey2.pdf

Mainprice, J., and Berenson, D., (2013, Nov) Human Robot Collaboration Manipulation Planning Using Early Prediction of Human Motion. Paper presented at the International Conference on Intelligent Robots and Systems, Tokyo, Japan.

Herman, A., Mauch, F., Fischnaller, K., Klemm, S., Roennau, A., and Dillmann, R. (2015, Sept.). Anticipate your Surroundings: Predictive Collision Detection between Dynamic Obstacles and Planned Robot Trajectories. Paper presented at the European Conference for Mobile Robots, Lincoln, UK.

Farin, G. and Hansford, D. (1999). Discrete Coons Patches. Computer Aided Geometry Design, 16, 691700.https://www.sciencedirect.com/science/article/abs/pii/S016783969900031X 